\title{
Remodeling of hippocampal mossy fibers is selectively induced seven days after the acquisition of a spatial but not a cued reference memory task
}

\author{
Jerome L. Rekart, ${ }^{1,5,7}$ C. Jimena Sandoval, ${ }^{1,6}$ Federico Bermudez-Rattoni, ${ }^{2}$ \\ and Aryeh Routtenberg ${ }^{1,3,4,7}$ \\ ${ }^{1}$ Department of Psychology, Northwestern University, Evanston, Illinois 60208, USA; ${ }^{2}$ Departamento de Neurociencias, Instituto \\ de Fisiologia Celular, Universidad Nacional Autonoma de Mexico, 04510 Mexico D.F., Mexico; ${ }^{3}$ Departments of Neurobiology \\ and Physiology and Neuroscience Institute, Northwestern University, Evanston, Illinois 60208, USA; ${ }^{4}$ Department of Physiology, \\ Feinberg School of Medicine, Northwestern University, Chicago, Illinois 60611, USA
}

\begin{abstract}
Relating storage of specific information to a particular neuromorphological change is difficult because behavioral performance factors are not readily disambiguated from underlying cognitive processes. This issue is addressed here by demonstrating robust reorganization of the hippocampal mossy fiber terminal field (MFTF) when adult rats learn the location of a hidden platform but not when rats learn to locate a visible platform. Because the latter task requires essentially the same behavioral performance as the former, the observed MFTF growth is seen as the consequence of specific input-dependent hippocampal activity patterns selectively generated by processing of extramaze but not intramaze cues. Successful performance on the hidden platform task requires formation of spatial memory. Increased MFTFs in hidden platform-trained rats are observed $7 \mathrm{~d}$ but not $2 \mathrm{~d}$ after training nor in swim controls. These results suggest that structural plasticity of the mossy fiber:CA3 circuit may contribute to the maintenance of long-lasting memory but not to the initial storage of the spatial context.
\end{abstract}

Does gross remodeling of anatomical circuits contribute to longterm memory storage? It has long been thought that the act of learning redefines the physical structure of neural connections (e.g., Ramon y Cajal 1905); however, there are few experimental demonstrations of such rearrangements.

Recently our group has provided direct evidence of learningdependent structural plasticity by demonstrating that training rats from two different strains (Holahan et al. 2006, 2007) to locate a hidden platform in a water maze induces growth of hippocampal granule cell mossy fiber terminal fields (MFTFs) from the stratum lucidum (SL) of CA3 into the stratum oriens (SO) and stratum pyramidale (SP). No growth was observed in swim controls that spent an equivalent period of time in the water maze as trained animals but with no platform present. When mice were trained in the same task no such growth was observed (Rekart et al. 2007).

The presence of hippocampal MFTF growth after spatial training in rats is convergent with data from lesion studies in rats (Morris et al. 1982; Sutherland et al. 1983; Moser et al. 1993, 1995) and mice (Logue et al. 1997), which suggests that spatial learning tasks, such as locating a hidden platform, are mediated by the hippocampus (O'Keefe and Nadel 1978; Eichenbaum 2000). Thus, it seems likely that growth of the mossy fibers consequent to spatial learning is driven by spatial learningdependent hippocampal activity.

One outstanding issue, however, is whether the learningdependent growth is truly specific for spatial (i.e., hippocampal-

Present addresses: ${ }^{5}$ Department of Psychology, Rivier College, Nashua, NH 03060, USA; ${ }^{6}$ Neurobiology Institute of the UNAM, Juriquilla, Queretaro 04510, Mexico.

${ }^{7}$ Corresponding authors.

E-mail jrekart@rivier.edu; fax (603) 897-8270.

E-mail aryeh@northwestern.edu; fax (847) 491-3557.

Article is online at http://www.learnmem.org/cgi/doi/10.1101//m.516507. dependent) learning, an issue raised in the original observations of Ramirez-Amaya et al. $(1999,2001)$. Although no growth was observed in swim control animals that were exposed to an equal amount of time in the pool as hidden platform-trained rats, swim controls, in contrast to trained animals, had no control over the amount of time spent in the pool. Indeed, the most reinforced behavior for swim control animals is likely the act of swimming toward experimenters for removal from the pool (Devan and McDonald 2001).

When learning the platform location in a water maze, the cognitive demands placed on the hippocampus can be reduced by including salient intramaze cues such as a visibly marked platform, which reduces the reliance on extramaze cues (Brandner and Schenk 1998). For example, rats with hippocampal lesions perform indistinguishably from sham controls when the location of the platform is clearly marked (Morris et al. 1982; Save and Poucet 2000). Thus, if the observed learning-dependent changes in MFTFs are truly caused by hippocampal-dependent spatial learning (Holahan et al. 2006), then the amount of hippocampal processing necessary should be increased during spatial processing relative to learning cued by a marked platform (Rudy et al. 1987). The present study tests whether such processing differences will be reflected in alterations in MFTF organization.

Male Wistar outbred rats (225-275 g; Charles River Laboratories) were group-housed (3/cage), fed ad libitum, and maintained on a 12-h light-dark cycle (lights on 06:00-18:00). Animals were handled for 3-5 d prior to behavioral training. Training took place during the light cycle (between 10:00-17:00), and all animal use and care were in accordance with Northwestern University Center for Comparative Medicine guidelines.

Rats were trained in the water maze similar to what has been described previously (Holahan et al. 2006). One modification to the Holahan et al. paradigm is that animals were randomly separated into three (rather than two) groups: In addition to those 
trained to find a hidden platform (HIDDEN; $10 \mathrm{~cm}$ in diameter submerged $2 \mathrm{~cm}$ beneath surface of water; $n=7$ ) and the swim control rats (SC; $n=7$ ) that were yoked to HIDDEN rats and spent the same amount of time in the pool (without a platform) as that animal, there were rats trained to find a visible platform (VISIBLE; platform $3 \mathrm{~cm}$ above the surface of the water and marked by a yellow Styrofoam buoy tethered to the platform; $n=7$ ). Rats learning the location of the hidden and visible platforms were trained for 10 trials/d using 10 start points for $5 \mathrm{~d}$. The same extra-maze cues were visible to all rats regardless of training condition. Animals were then sacrificed after either a 2- or a 7-d "incubation" period after the last training trial. Immediately before they were sacrificed, rats given $7 \mathrm{~d}$ of "incubation" were allowed to search a pool without a platform for $60 \mathrm{sec}$; the time spent in each quadrant and the number of platform crossings was recorded (probe test) using the HVS Image system (HVS) and analyzed using the public domain Wintrack system (D. Wolfer; http://www.dpwolfer.ch/wintrack/Index.htm).

Despite exhibiting similar rates of acquisition and latencies to reach the platform, the HIDDEN and VISIBLE rats exhibited a

A

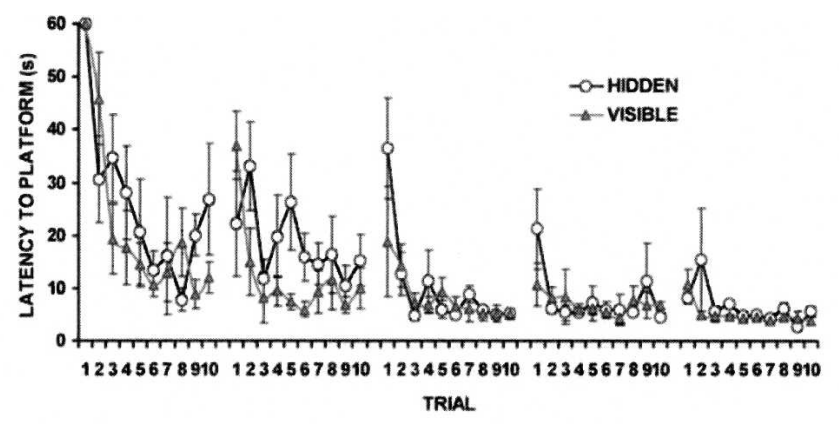

B
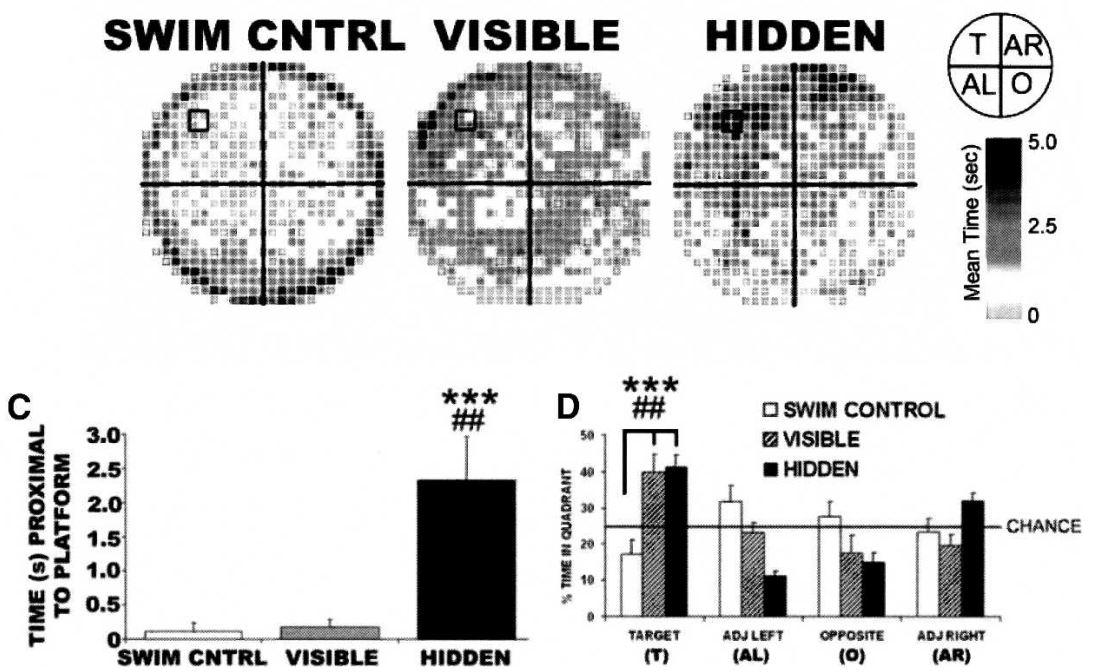

Figure 1. (A) Wistar rats trained to find a hidden (HIDDEN) or a visible (VISIBLE) platform display similar levels of acquisition after $5 \mathrm{~d}$ of training. (B) Occupancy plots from a 7-d probe test demonstrate that HIDDEN spent more time proximal to the location where the absent platform was previously located during training (square) than VISIBLE. Color coding is equivalent to the average time spent in each pixel $\left(\sim 48 \mathrm{~cm}^{2} /\right.$ pixel), with red indicating a maximum and deep blue indicating no time spent in a given portion of the pool. (C) Statistical analysis of occupancy plots showed that HIDDEN rats spent significantly more time within an area (annulus $=$ platform +1 platform diameter) proximal to the original platform location than either VISIBLE or yoked swim control (SWIM CNTRLS) rats. ${ }^{* * *} P=0.005$ vs. SWIM CNTRL; \#\#P<0.025 vs. VISIBLE. (D) HIDDEN (Tukey post-hoc, ${ }^{* * *} P<0.0025$ ) and VISIBLE (Tukey post-hoc \#\#P<0.01) rats spent significantly more time in the target quadrant than swim controls; HIDDEN rats spent significantly more time than predicted by chance in the target quadrant ( $T$; 1 -sample t-test $\left.t_{(4)}=4.99, P<0.005\right)$. Values are represented as mean \pm SEM. (AR) Adjacent right quadrant; (AL) adjacent left quadrant; $(\mathrm{O})$ opposite quadrant. (A color version of Figure $1 \mathrm{~B}$ is available upon request.) significant difference in 1-wk retention (Fig. 1A), as shown in the occupancy plots of Figure 1, B and C. Occupancy plots (Fig. 1B) from this 1 -wk probe test revealed the greater accuracy of the to VISIBLE and SC rorm location exhibited by HIDDEN relative the than VISIBLE and SC rats in the region of the pool proximal

Mossy fiber remodeling was assessed using hippocampal tissue from trained rats that was prepared, stained, and analyzed using the "split-brain" method detailed in Holahan et al. (2006) sected, and corongl $4 \%$ paraformaldehyde. It was sectioned at $40 \mu \mathrm{m}$ in $t$ coronal plane and every fifth section was systematically chosen from a randomly chosen starting point at the extreme septal pole us for a total of six sections/animal. For immunohistochemical analyses, the other hemisphere was fixed in $4 \%$ paraformaldehyde. For Timm's analysis, digital images of the entire regio inferior were obtained using an Olympus BX61 microscope with a DP70 camera $(12.5$ megapixels, Olympus). Images were then coded and a point-counting grid (squares $=25 \mu \mathrm{m} /$ side) was digitally superimposed. Grid intersections overlying dark Timm's silver precipitate were counted as belonging either to the SL or to the strata oriens and stratum pyramidale (SOSP) using described criteria (Holahan et al. 2006). The areas for the SO and SP were combined as they are hard to distinguish, particularly proximal to CA3c (Claiborne et al. 1986). Comparisons between groups were made using the ratio of the estimated area in SOSP to that in SL to account for any size differences between individual animals. Subsets of Timm's counts were conducted by two independent raters using identical selection criteria. The inter-rater reliability was found to be significantly similar $(P<0.0001)$ with a Pearson correlation coefficient of $r=+0.95$.

The difference in the quantitative level of precision of the memory for the target position was linked to both a qualitative and a quantitative difference in MFTF staining. Specifically, there was a striking, readily discernible elevation of mossy fiber presence in the SO of HIDDEN that was not observed in VISIBLE or indeed SC (Fig. 2A).

Quantitative analysis showed that the normalized area of Timm's staining (e.g., divided by area of SL) in the CA3 SOSP of HIDDEN rats sacrificed $7 \mathrm{~d}$ after training was significantly greater than that of VISIBLE rats (Fig. 2B; Tukey posthoc, $P<0.025)$ and SC rats (Tukey posthoc, $P<0.01)$. In VISIBLE rats there was 
A
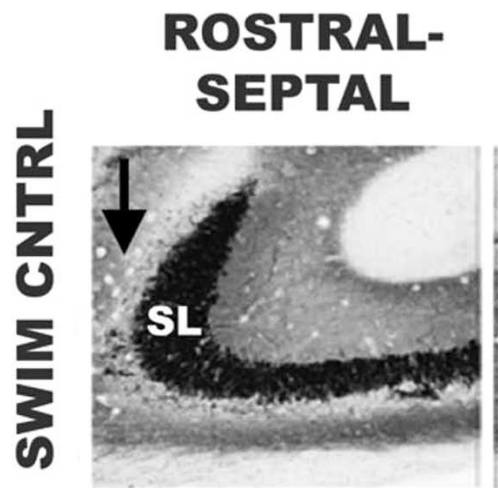

MID-SEPTAL
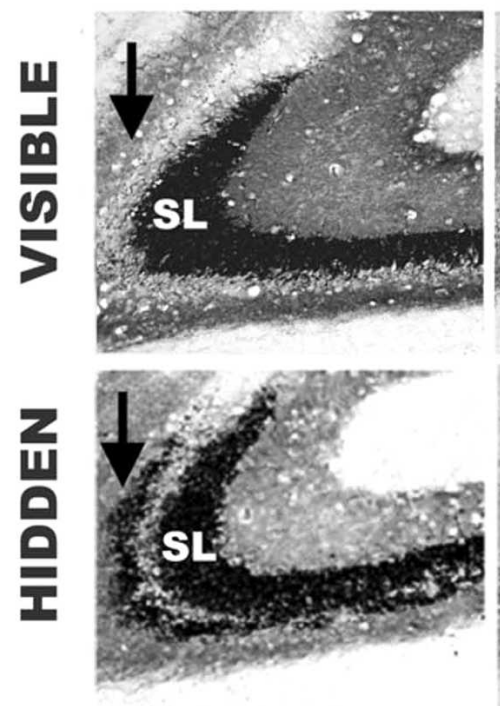
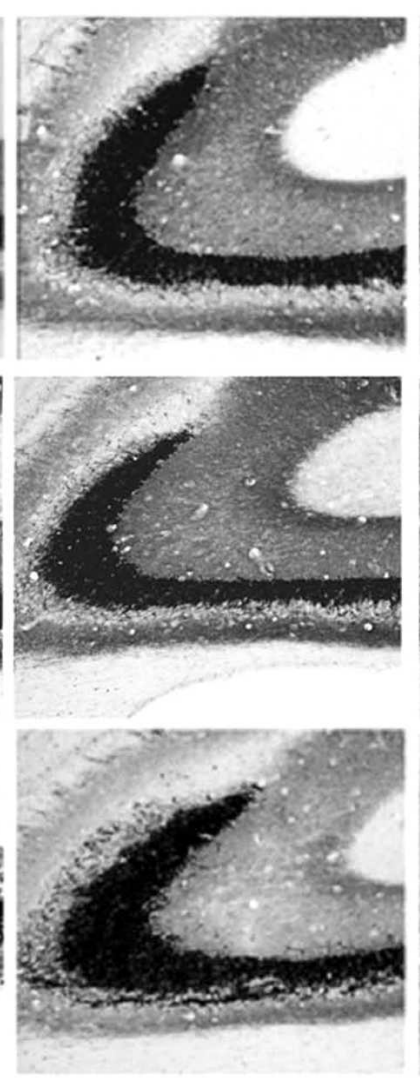

\section{CAUDAL- SEPTAL}
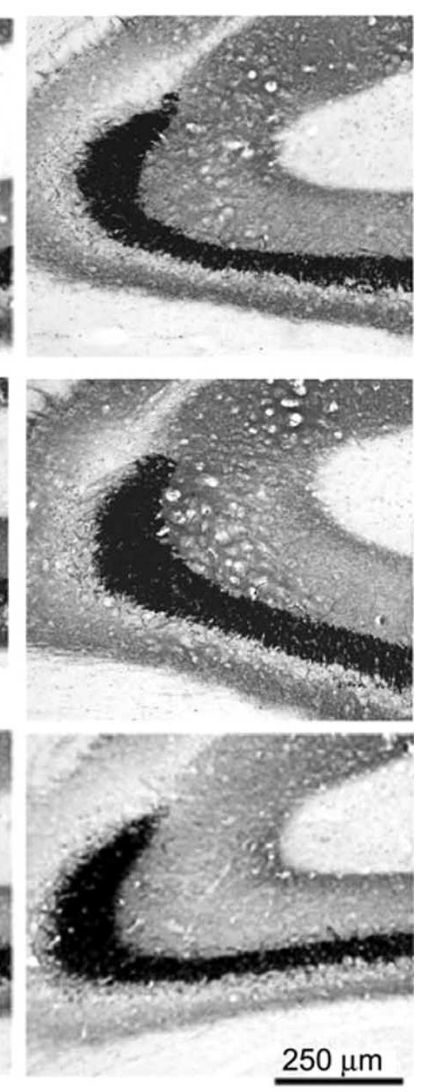

B TOTAL CA3

C

CA3b ONLY
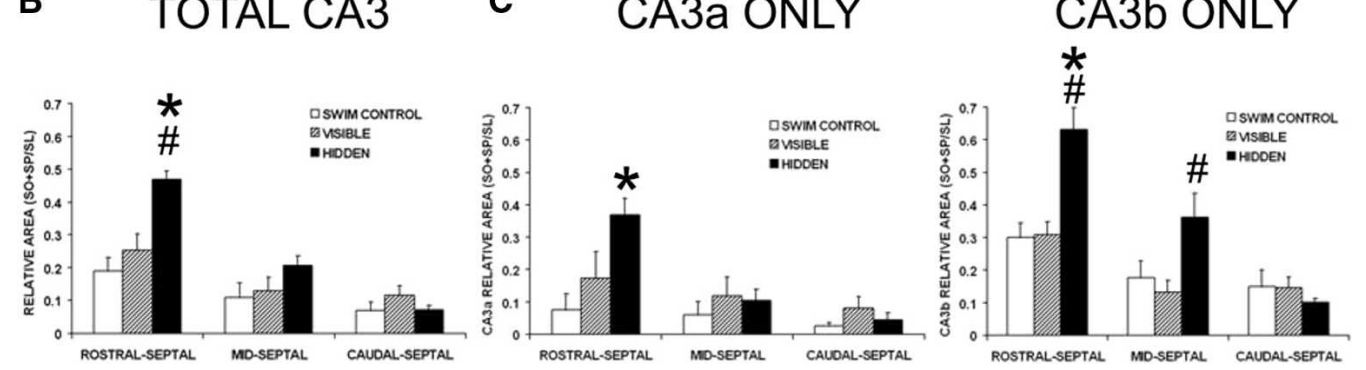

Figure 2. Selective learning-induced mossy fiber terminal field expansion is observed after hidden platform training but is not observed in visibleplatform-trained rats. (A) Seven days after the end of training, robust increases in Timm's heavy metal staining, indicative of zinc-containing hippocampal mossy fiber axons and terminals, were only observed in the strata oriens and pyramidale (SOSP; arrows) of hidden platform-trained rats ( $n=4$ /group). This increment was restricted to the rostral-most septal hippocampus (ROSTRAL-SEPTAL) and was less apparent posteriorly (MID-SEPTAL) with no observable differences evident in the most posterior third of the septal hippocampus (CAUDAL-SEPTAL). (SL) Stratum lucidum. ${ }^{*} P<0.025$ vs. SWIM CNTRL; $\# P<0.05$ vs. VISIBLE. (B) The relative area of Timm's staining in the SOSP of the rostral-septal hippocampus was significantly greater in HIDDEN rats than in visible platform-trained (VISIBLE) or swim control (SWIM CONTROL) rats. (C) Significant hidden platform training-induced expansions are observed in the rostral- and mid-septal SOSP of CA3b and the rostral-septal SOSP of CA3a.

no significant difference in the proportion of SOSP MFTFs relative to swim controls (Tukey post-hoc, $P=0.670$ ). Absolute SL area was not found to differ significantly between training conditions. Moreover, the significant training-dependent increments in the SOSP of HIDDEN rats were restricted to the rostralmost third of the septal hippocampus (Fig. 2A), a point emphasized by both Ramirez-Amaya et al. (2001) and Holahan et al. (2006).

In rats sacrificed $48 \mathrm{~h}$ after training, the size and relative distribution of MFTFs did not differ as a function of training. Specifically, no significant effect of training on the size of the
SOSP, the SL, or the SOSP/SL within the regio inferior was found (data not shown).

In rats sacrificed $7 \mathrm{~d}$ after training, a subregional analysis of the reorganization of the MFTFs $7 \mathrm{~d}$ after training revealed significant local remodeling. The total CA3 area was divided into component CA3a and CA3b subregions; the former contains collateral branches from parent mossy fibers in the distal SL while the latter contains terminals originating with the infra- and intrapyramidal mossy fiber (IIPMF) projections (Swanson et al. 1978). We differentiated between regions by designating any Timm's precipitate located dorsal to a virtual dividing line placed 
on the border of the SL and SP as belonging to CA3a. CA3b encompassed all remaining Timm's precipitate located ventral to and in contact with the virtual dividing line. CA3c was located within the blades of the granule cell layer and was excluded from the subregional analysis.

With this parcellation of hippocampal subfields, one observes significant increments in the normalized size of the SOSP of HIDDEN rats relative to yoked SCs in CA3a (Tukey post-hoc, $P<0.025$ ) and CA3b (Tukey post-hoc, $P<0.025$ ) that is greater than VISIBLE rats (Tukey post-hoc, $P<0.05$ ) in CA3b (Fig. 2C). These results suggest that the increased number of mossy fiber terminals suggested by Timm's staining are most likely to originate from both existing mossy fibers in the IIPMF pathway (CA3b) and, as has been previously described (Holahan et al. 2006), the distal suprapyramidal mossy fibers (CA3a).

We used two antibodies to determine whether learningspecific changes in Timm's staining were due to an increased distribution of Timm's precipitate into existing mossy fiber axons or represented a growth or remodeling of these axons (Holahan et al. 2006). The antibody specific for zinc transporter 3 (ZnT3) preferentially and selectively stains mossy fibers and its terminals (Palmiter et al. 1996) and an antibody against Tau1, which is restricted to axons and has served as a marker for axonal growth (Binder et al. 1985; Caceres and Kosik 1990), were used. As shown in Figure 3, hidden platform training did indeed in-
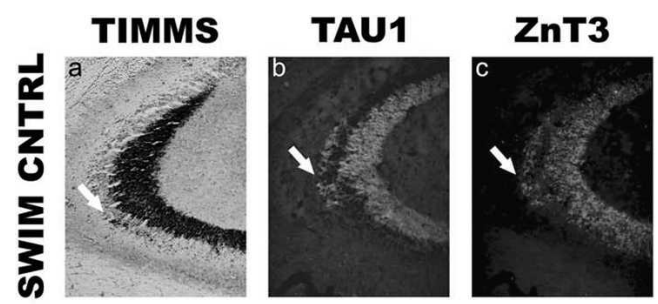

MERGE
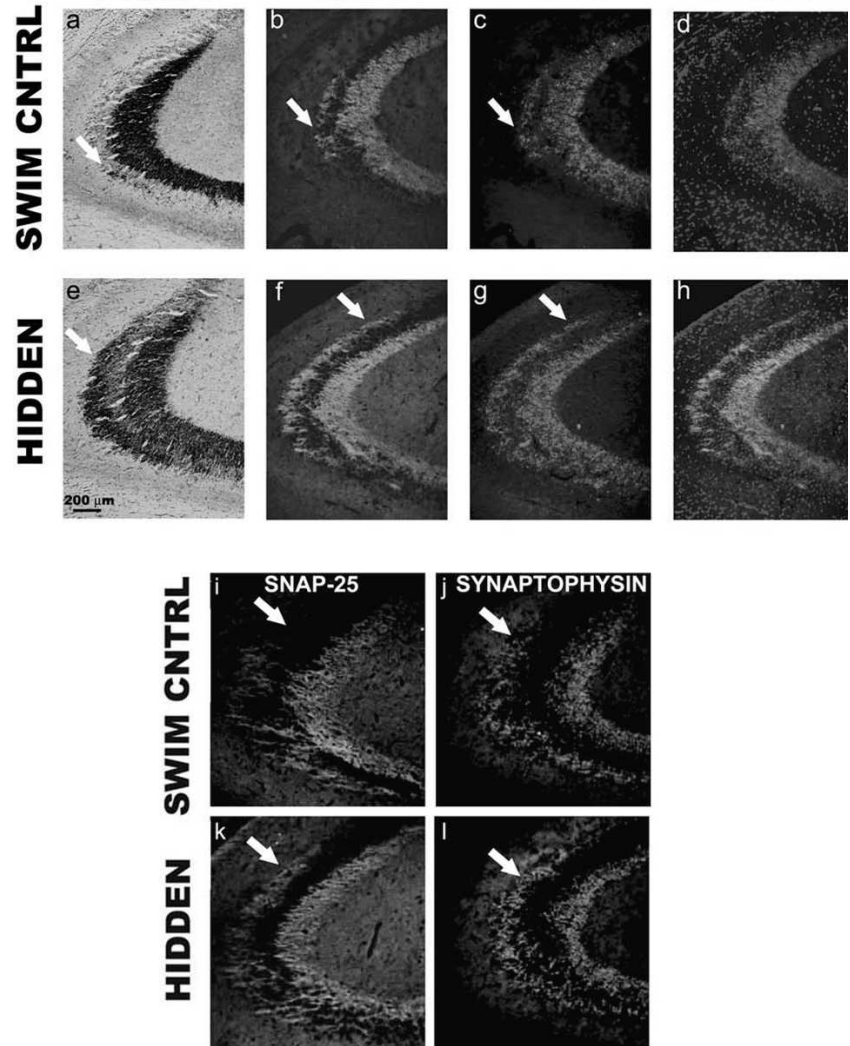

Figure 3. Increased immunoreactivity for zinc transporter $(g)$ and the axonal protein tau $(f)$ are observed in the rostral-septal SOSP of hidden platform-trained rats. Coronal sections from the other hemisphere was obtained from HIDDEN and SWIM CNTRL rats and were processed for Timm's staining (TIMMS), tau1, ZnT3, or tau1 + ZnT3 staining with a blue DAPI nuclear counterstain (MERGE). In addition, immunoreactivity for synaptic markers SNAP-25 $(i, k)$ and synaptophysin $(j, D)$ is increased in the rostral-septal SOSP of hidden platform-trained $(k, l)$ but not swim control $(i, j)$ rats. (A color version of Figure 3 is available upon request.) crease the distribution of ZnT3 (Fig. 3). Importantly, increased staining for tau1 was also observed in the CA3 SOSP of trained animals (Fig. 3). Moreover, both the axonal Tau1 and the mossy fiber-specific ZnT3 in the CA3 SOSP of trained animals were colocalized within the MFTF (see MERGE in Fig. 3), strongly supporting the view that training induced a new growth or remodeling of mossy fibers. To assess whether the increases in Tau1 and ZnT3 were associated with concomitant training-dependent increases in proteins associated with functional synapses, we also assessed immunoreactivity for the presynaptic markers synaptophysin (Sudhof and Jahn 1991) and SNAP-25 (Kretzschmar et al. 1996). As panels i-l of Figure 3 demonstrate, hidden platform training resulted in qualitative increments in the immunoreactivity for both SNAP-25 and synaptophysin in the CA3 SOSP.

The present results show that spatial but not cued learning can act as an input-dependent trigger for remodeling of mossy fibers and their terminal arbor. The lack of an effect of cued training using the visible platform on MFTF growth suggests that merely learning the location of a platform is not sufficient to induce MFTF structural plasticity. Thus, only when the task requires the processing of spatial and contextual cues to locate the platform, a function which is known to selectively activate hippocampal granule cell activity (Jung and McNaughton 1993), does MFTF growth occur. Such selective hippocampal activation may in turn preferentially recruit growth-associated and cytoskeletal proteins that mediate the morphological alterations in the mossy fiber system. Indeed, positive immunoreactivity for two proteins, one for the mossy fiber-containing zinc transporter and one for a marker of the axonal cytoskeleton, suggests that new growth or expansion of mossy fibers has occurred. That there may also be presynaptic terminal proliferation is suggested by training-induced elevated staining by the presynaptic markers synaptophysin and SNAP-25 of the SO. Behavioral and physiological evidence of other authors as well as the current results support the localization of function to the anterior portions of the septotemporal axis of the hippocampus. For example, the restriction of growth to the rostral third of the septal hippocampus of hidden platform-trained rats observed in the present study is consistent with findings that lesions of the septal but not the temporal hippocampus produce specific impairments on tasks requiring spatial processing (Moser et al. 1993, 1995; Hock and Bunsey 1998; Bannerman et al. 1999).

Septotemporal behavioral dissociations likely arise from corresponding differences in the organization of projections to the hippocampus. For example, neurons from the dorsolateral band of the medial entorhinal cortical area, in layer II, which project to granule cells in the septal hippocampus (Ruth et al. 1988; Dolorfo and Amaral 1998), show spatial selectivity, but cells in the ventromedial band, which provide input to the temporal hippocampus, do not (Fyhn et al. 2004).

Within the hippocampus, the mossy fibers have been shown to be critical for spatial learning as chelation of zinc from hippocampal mossy fibers impairs spatial learning in rats (Frederickson et al. 1990). More generally, rats with hippocampal lesions are impaired in finding a hidden platform but perform as well as sham controls if the platform location is visibly marked (Morris et al. 1982). Consistent with this idea, the probe-test occupancy plot of visible platform-trained rats clearly demonstrated that there is little more than a general sense (e.g., "gist"; Koriat et al. 2000) as to exact platform location. In contrast, the results from the hidden platform-trained rats suggest the formation of a strong spatial representation for the exact spatial coordinates of the hidden platform (see Fig. 1B), which may be related to the increased network complexity brought about by MFTF expansion. Because the initial learning over the 5-d training period produces no significant growth $48 \mathrm{~h}$ after training, 
mossy fiber expansion is more likely to play a role in those processes involved in long-lasting or remote spatial memory rather than early stages of information processing.

Is there a potential contribution of neurogenesis to the learning-induced mossy fiber terminal growth observed in the present study? If so, the observed expansion could be attributed to the extension and ramification of the axonal arbor of recently born dentate gyrus granule cells, as spatial but not cued learning in the water maze has been shown to increase nascent-born granule cell survival (Gould et al. 1999). Indeed, 4 to $10 \mathrm{~d}$ are required after mitosis for adult-derived granule cells to extend axons into the distal CA3 MFTFs (Hastings and Gould 1999). Because learning-dependent mossy fiber growth in this report requires 3-7 d after training to be observed, the roughly similar timelines for the growth observed here and that due to neurogenesis are intriguing.

Regardless of the age of the granule cells contributing to the MFTF expansion, it is notable that the outgrowth does not occur during the $5 \mathrm{~d}$ training period nor is it detectable $2 \mathrm{~d}$ after training. Thus, only 3-7 d after the termination of training does the growth of the axon terminals become manifest. Importantly, this occurs during the 7-d post-training period at a time when the animal is no longer exposed to the training apparatus, experimental room, or experimenters. Thus, the growth process occurs off-line and, as has been previously shown, persists for at least 30 d (Ramirez-Amaya et al. 2001).

The cellular processes that occur during the 7-d posttraining home cage "incubation" period may instead reflect the involvement of endogenous neural rehearsal mechanisms that are part of the off-line processing in networks that participate in the representation of the spatial memory (Santini et al. 2001; Hoffman and McNaughton 2002; Routtenberg and Rekart 2005) and that can be observed in awake, freely moving animals (Foster and Wilson 2006). Indeed, the current results are entirely consistent with the recently proposed model of memory that specifies "cryptic rehearsal" or off-line processing as critical for maintaining post-translation modifications after learning has taken place (Routtenberg and Rekart 2005). These continuing off-line neural activity patterns are considered necessary for regulating learning-induced post-translational modification of proteins that sustain long-lasting memory. Studying the molecular and physiological events during the 7 -d post-training incubation period will doubtless prove of value in testing the neural and molecular growth-promoting events that occur after the learning trials have ceased.

\section{Acknowledgments}

This work was supported by an NIMH traineeship (MH TG 067564) to J.L.R. and by research grants from NSF (980090723) and NIMH (MH65436) to A.R.

\section{References}

Bannerman, D.M., Yee, B.K., Good, M.A., Heupel, M.J., Iversen, S.D., and Rawlins, J.N. 1999. Double dissociation of function within the hippocampus: A comparison of dorsal, ventral, and complete hippocampal cytotoxic lesions. Behav. Neurosci. 113: 1170-1188.

Binder, L.I., Frankfurter, A., and Rebhun, L.I. 1985. The distribution of tau in the mammalian central nervous system. J. Cell Biol. 101: 1371-1378.

Brandner, C. and Schenk, F. 1998. Septal lesions impair the acquisition of a cued place navigation task: Attentional or memory deficit? Neurobiol. Learn. Mem. 69: 106-125.

Caceres, A. and Kosik, K.S. 1990. Inhibition of neurite polarity by tau antisense oligonucleotides in primary cerebellar neurons. Nature 343: $461-463$.

Claiborne, B.J., Amaral, D.G., and Cowan, W.M. 1986. A light and electron microscopic analysis of the mossy fibers of the rat dentate gyrus. J. Comp. Neurol. 246: 435-458.
Devan, B.D. and McDonald, R.J. 2001. A cautionary note on interpreting the effects of partial reinforcement on place learning performance in the water maze. Behav. Brain Res. 119: 213-216.

Dolorfo, C.L. and Amaral, D.G. 1998. Entorhinal cortex of the rat: Topographic organization of the cells of origin of the perforant path projection to the dentate gyrus. J. Comp. Neurol. 398: 25-48.

Eichenbaum, H. 2000. Hippocampus: Mapping or memory? Curr. Biol. 10: R785-R787.

Foster, D.J. and Wilson, M.A. 2006. Reverse replay of behavioural sequences in hippocampal place cells during the awake state. Nature 440: $680-683$.

Frederickson, R.E., Frederickson, C.J., and Danscher, G. 1990. In situ binding of bouton zinc reversibly disrupts performance on a spatial memory task. Behav. Brain Res. 38: 25-33.

Fyhn, M., Molden, S., Witter, M.P., Moser, E.I., and Moser, M.B. 2004. Spatial representation in the entorhinal cortex. Science 305: $1258-1264$.

Gould, E., Beylin, A., Tanapat, P., Reeves, A., and Shors, T.J. 1999. Learning enhances adult neurogenesis in the hippocampal formation. Nat. Neurosci. 2: 260-265.

Hastings, N.B. and Gould, E. 1999. Rapid extension of axons into the CA3 region by adult-generated granule cells. J. Comp. Neurol. 413: $146-154$.

Hock Jr., B.J. and Bunsey, M.D. 1998. Differential effects of dorsal and ventral hippocampal lesions. J. Neurosci. 18: 7027-7032.

Hoffman, K.L. and McNaughton, B.L. 2002. Sleep on it: Cortical reorganization after-the-fact. Trends Neurosci. 25: 1-2.

Holahan, M.R., Rekart, J.L., Sandoval, J., and Routtenberg, A. 2006. Spatial learning induces presynaptic structural remodeling in the hippocampal mossy fiber system of two rat strains. Hippocampus 16: $560-570$.

Holahan, M.R., Honegger, K.S., and Routtenberg, A. 2007. Expansion and retraction of hippocampal mossy fibers during post-weaning development: Strain-specific effects of NMDA receptor blockade. Hippocampus 17: 58-67.

Jung, M.W. and McNaughton, B.L. 1993. Spatial selectivity of unit activity in the hippocampal granular layer. Hippocampus 3: 165-182.

Koriat, A., Goldsmith, M., and Pansky, A. 2000. Toward a psychology of memory accuracy. Annu. Rev. Psychol. 51: 481-537.

Kretzschmar, S., Volknandt, W., and Zimmermann, H. 1996. Colocalization on the same synaptic vesicles of syntaxin and SNAP-25 with synaptic vesicle proteins: A re-evaluation of functional models required? Neurosci. Res. 26: 141-148.

Logue, S.F., Paylor, R., and Wehner, J.M. 1997. Hippocampal lesions cause learning deficits in inbred mice in the Morris water maze and conditioned-fear task. Behav. Neurosci. 111: 104-113.

Morris, R.G., Garrud, P., Rawlins, J.N., and O'Keefe, J. 1982. Place navigation impaired in rats with hippocampal lesions. Nature 297: 681-683.

Moser, E., Moser, M.B., and Andersen, P. 1993. Spatial learning impairment parallels the magnitude of dorsal hippocampal lesions, but is hardly present following ventral lesions. J. Neurosci. 13: 39163925.

Moser, M.B., Moser, E.I., Forrest, E., Andersen, P., and Morris, R.G. 1995. Spatial learning with a minislab in the dorsal hippocampus. Proc. Natl. Acad. Sci. 92: 9697-9701.

O'Keefe, J. and Nadel, L. 1978. The hippocampus as a cognitive map. Clarendon Press, London, UK.

Palmiter, R.D., Cole, T.B., Quaife, C.J., and Findley, S.D. 1996. ZnT-3, a putative transporter of zinc into synaptic vesicles. Proc. Natl. Acad. Sci. 93: 14934-14939.

Ramirez-Amaya, V., Escobar, M.L., Chao, V., and Bermudez-Rattoni, F. 1999. Synaptogenesis of mossy fibers induced by spatial water maze overtraining. Hippocampus 9: 631-636.

Ramirez-Amaya, V., Balderas, I., Sandoval, J., Escobar, M.L., and Bermudez-Rattoni, F. 2001. Spatial long-term memory is related to mossy fiber synaptogenesis. J. Neurosci. 21: 7340-7348.

Ramon y Cajal, S. 1905. Histology of the nervous system. Oxford University Press, New York.

Rekart, J.L., Sandoval, J., and Routtenberg, A. 2007. Learning-induced axonal remodeling: Evolutionary divergence and conservation of two components of the mossy fiber system within Rodentia. Neurobiol. Learn. Mem. 87: 225-235.

Routtenberg, A. and Rekart, J.L. 2005. Post-translational protein modification as the substrate for long-lasting memory. Trends Neurosci. 28: 12-19.

Rudy, J.W., Stadler-Morris, S., and Albert, P. 1987. Ontogeny of spatial navigation behaviors in the rat: Dissociation of "proximal"- and "distal"-cue-based behaviors. Behav. Neurosci. 101: 62-73.

Ruth, R.E., Collier, T.J., and Routtenberg, A. 1988. Topographical relationship between the entorhinal cortex and the septotemporal 
axis of the dentate gyrus in rats: II. Cells projecting from lateral entorhinal subdivisions. J. Comp. Neurol. 270: 506-516.

Santini, E., Muller, R.U., and Quirk, G.J. 2001. Consolidation of extinction learning involves transfer from NMDA-independent to NMDA-dependent memory. J. Neurosci. 21: 9009-9017.

Save, E. and Poucet, B. 2000. Involvement of the hippocampus and associative parietal cortex in the use of proximal and distal landmarks for navigation. Behav. Brain Res. 109: 195-206.

Sudhof, T.C. and Jahn, R. 1991. Proteins of synaptic vesicles involved in exocytosis and membrane recycling. Neuron 6: 665-677.
Sutherland, R.J., Whishaw, I.Q., and Kolb, B. 1983. A behavioural analysis of spatial localization following electrolytic, kainate- or colchicine-induced damage to the hippocampal formation in the rat. Behav. Brain Res. 7: 133-153.

Swanson, L.W., Wyss, J.M., and Cowan, W.M. 1978. An autoradiographic study of the organization of intrahippocampal association pathways in the rat. J. Comp. Neurol. 181: 681-715.

Received December 28, 2006; accepted in revised form May 1, 2007. 


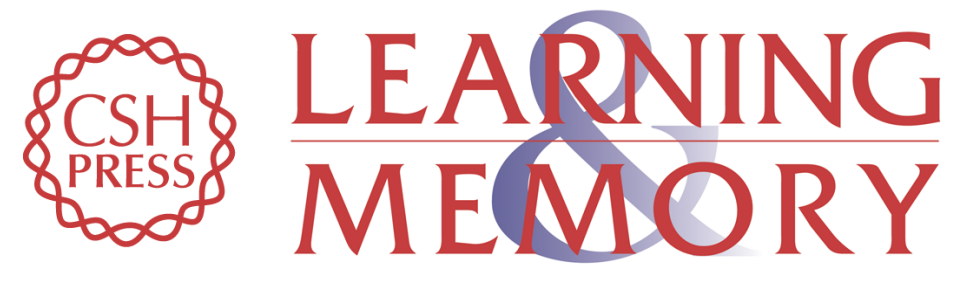

\section{Remodeling of hippocampal mossy fibers is selectively induced seven days after the acquisition of a spatial but not a cued reference memory task}

Jerome L. Rekart, C. Jimena Sandoval, Federico Bermudez-Rattoni, et al.

Learn. Mem. 2007, 14:

Access the most recent version at doi:10.1101//m.516507

References This article cites 36 articles, 8 of which can be accessed free at: http://learnmem.cshlp.org/content/14/6/416.full.html\#ref-list-1

License

Email Alerting

Receive free email alerts when new articles cite this article - sign up in the box at the Service top right corner of the article or click here. 